\title{
Poisoning due to ingestion of amatoxin-containing mushrooms in South Korea: a systematic review and meta-analysis
}

\author{
Chiwon Ahn ${ }^{1}$, Hyunggoo Kang ${ }^{2, *}$, Tae Ho Lim², Jaehoon Oh²
}

${ }^{1}$ Department of Emergency Medicine, College of Medicine, Chung-Ang University, Seoul, 06974, South Korea ${ }^{2}$ Department of Emergency Medicine, College of Medicine, Hanyang University, Seoul, 04763, South Korea

\section{*Correspondence}

emer0905@gmail.com

(Hyunggoo Kang)

\begin{abstract}
Objective: Poisoning caused by the ingestion of amatoxin-containing mushrooms is lifethreatening and requires urgent attention. However, only few studies have evaluated the factors that predict mortality owing to mushroom poisoning. We conducted a systematic review of amatoxin poisoning in South Korea and meta-analysis of the association between severe early-stage central nervous system symptoms and mortality in cases of amatoxin poisoning.

Methods: The Embase, MEDLINE, Web of Science, KMbase, and Korean Studies Information System databases were searched for articles up to July 2020. We included case reports, case series, and observational studies on Amanita poisoning in South Korea. Outbreak area, incubation time, clinical course, management, and outcomes were evaluated. We then conducted a meta-analysis of the association between severe central nervous system symptoms and mortality.

Results: Sixteen articles were included in the review and five in the meta-analysis. Outbreaks occurred principally in Gyeongbuk and in the western part of Gangwon. All patients had gastrointestinal symptoms, such as watery diarrhea. Liver failure occurred 2-3 days after mushroom intake in most patients, and ingestion of amatoxins from mushroom consumption was associated with high mortality risk. The risk of mortality among patients with altered mental status or seizures was 10 times higher than that among patients without these symptoms (risk ratio $=10.56,95 \%$ confidence interval $=2.73$ 40.83).

Conclusions: Amanita mushrooms are often mistaken for edible mushrooms, and their ingestion is frequently fatal. Aggressive treatment must be pursued in patients with severe central nervous system symptoms, such as altered mental status or seizures.
\end{abstract}

\section{Keywords}

Amanita; Amatoxins; Mushrooms; Mushroom poisoning

\section{Introduction}

Mushrooms are the "fruiting bodies" (reproductive structures) of fungi that produce spores. Mushrooms grow out of the ground or wood. There are over 5,000 species of mushrooms worldwide. Of these, 50 to 100 are poisonous [1-3], and individuals often seek medical attention after ingesting wild poisonous mushrooms, which are mistaken for edible ones. Although accidental mushroom poisoning occurs regularly, individuals lack knowledge regarding the type of mushroom they have ingested in $85-95 \%$ of cases, and poisoning is usually recognized only through the development of clinical symptoms after intake [1, 4]. Accurate information regarding which mushrooms have been ingested is needed to establish a treatment plan; however, those who have ingested mushrooms often do not bring a preserved specimen to the medical institution where they are receiving treatment, and testing is time- consuming even when specimens are available [4].

The genus Amanita is the most lethal group of poisonous mushrooms worldwide, with Amanita phalloides accounting for more than $90 \%$ of mushroom poisoning fatalities [5]. Amatoxin poisoning is the most common cause of mushroom poisoning globally, and Amanita virosa and Amanita subjunquillea are the most common poisonous mushrooms [6]. Amatoxin poisoning can cause liver or renal failure, and patients generally present to the hospital in a critical condition because of the long incubation period; external signs and symptoms are generally not visible until the disease is well advanced $[1,7,8]$. The toxin acts on various organs, causing several clinical symptoms, and the development of kidney failure, including irreversible liver damage, leads to death.

In addition, since multi-organ failure can progress rapidly, it requires quick and intensive care support from the early phase 


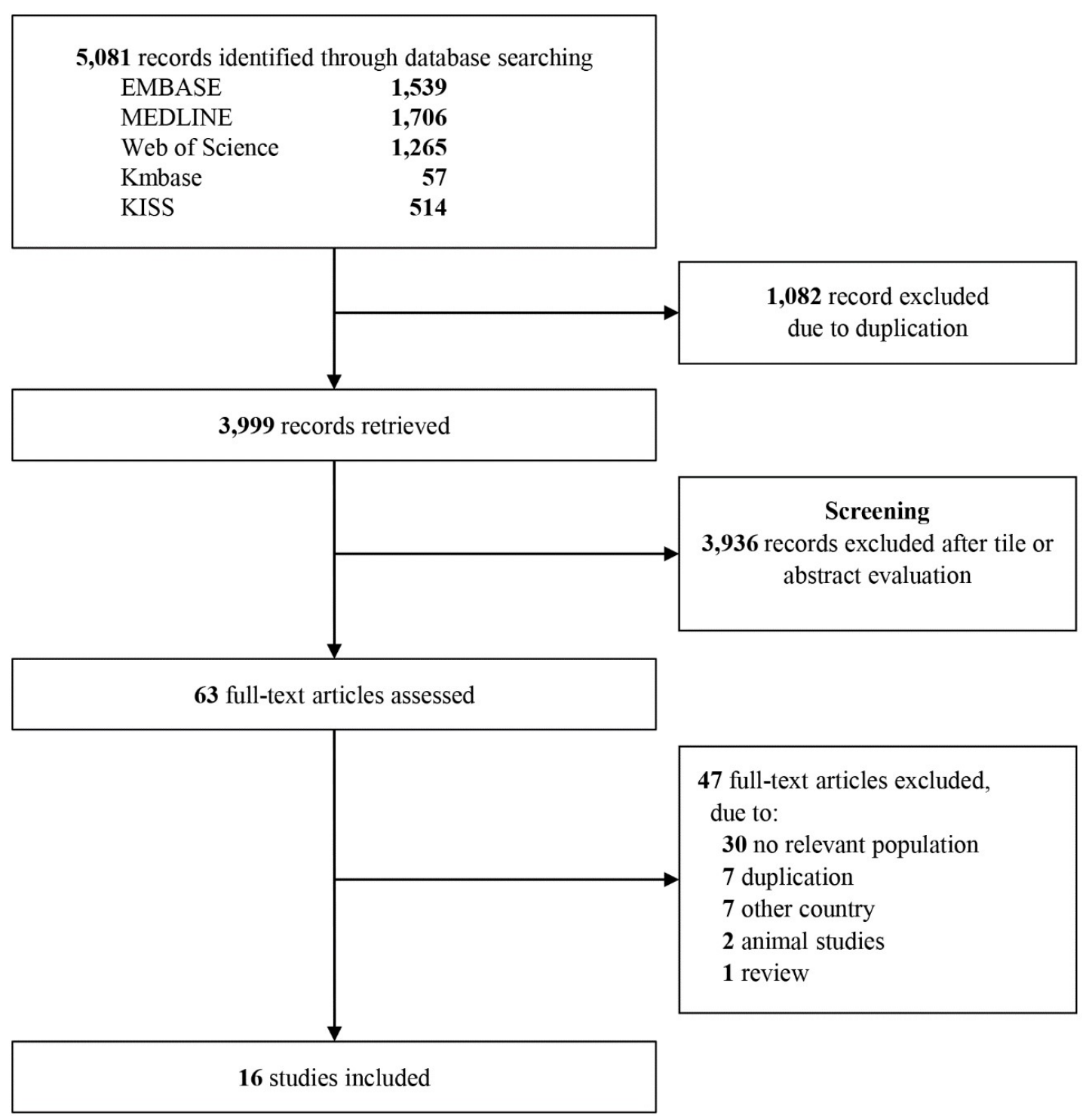

F I G U R E 1. Flow chart of study selection.

in emergency room.

In the present study, we will investigate cases of amatoxin poisoning in South Korea, and evaluate the clinical manifestations and intensive care for the severe symptoms of poisoning. In addition, a meta-analysis of the increase in mortality when amatoxin-poisoning patients initially developed severe neurological symptoms will be performed.

\section{Materials and methods}

The outcomes of this study were calculated in a meta-analysis of the research question: "Are severe early-stage CNS symptoms associated with increased mortality in cases of amatoxin poisoning?"

\subsection{Search strategy and data sources}

We searched the MEDLINE, Embase, and Web of Science databases for literature published in academic journals outside Korea, and KoreaMED, Korean Studies Information System, and KMbase for literature published in Korean academic journals up to July 2020. The following search keywords were selected after discussion with all authors: "Amanita", "Amatoxin", "mushroom poisoning", and mixed terms generated from these ones (See also Table S1).

\subsection{Study selection and data extraction}

Selected studies were entered into the reference management software Endnote 9.3 (Thomson Reuters, New York, NY, USA). Duplicate articles were excluded after comparing the title, authors, year of publication, and journal of all identified studies. We then excluded reviews, editorials, letters, conference proceedings, comments, and articles that were not in English or Korean. Three authors (CA, JL, and HS) screened each article and checked the title, abstract, and article type. We then selected studies on mushroom poisoning for full-text review. We also reviewed the reference lists of the selected articles to include additional articles in the review. Data from all included studies were extracted using Microsoft Excel 2016 (Microsoft, Redmond, WA, USA). 
TABLE 1. Details of the included studies

\begin{tabular}{|c|c|c|c|c|c|c|c|c|}
\hline Study & No. & Location & Type of mushroom & $\begin{array}{l}\text { Incubation } \\
\text { time }\end{array}$ & Initial symptom & Main problem & Intervention & Outcome \\
\hline Hyun [11] (1973) & 1 & Gyeonggi & Amanita genus & 2 & $\begin{array}{l}\text { GI symptoms Confusion, } \\
\text { Jaundice }\end{array}$ & Liver failure & Liver biopsy & Death \\
\hline Lee [12] (1990) & 16 & $\begin{array}{l}\text { The Western part of } \\
\text { Gangwon }\end{array}$ & Amanita genus & 14 & $\begin{array}{l}\text { Altered mental state } \\
\text { (drowsy 3, stupor } 2, \\
\text { semicoma } 1, \text { coma } 1 \text { ) }\end{array}$ & $\begin{array}{ll}\text { Liver } & \text { failure, } \\
\text { Coagulopathy } & \end{array}$ & $\begin{array}{l}\text { Charcoal intake (3), } \\
\text { Hemodialysis (2) }\end{array}$ & $\begin{array}{l}6 \text { improved, } 5 \\
\text { deaths, } 1 \text { DOA, } 4 \\
\text { poor outcome }\end{array}$ \\
\hline Lee [13] (1990) & 1 & - & Amanita virosa & 12 & $\begin{array}{l}\text { GI symptoms, Anuria } \\
\text { (late) }\end{array}$ & $\begin{array}{l}\text { Liver failure } \\
\text { phase), Renal farly } \\
\text { (late phase) }\end{array}$ & Renal biopsy & Improved \\
\hline Ahn [14] (1993) & 2 & Chungbuk & Amanita virosa & 9 & GI symptoms & $\begin{array}{l}\text { Liver failure, Renal fail- } \\
\text { ure (1 Pt.) }\end{array}$ & $\begin{array}{l}\text { Gastric lavage, Charcoal } \\
\text { intake, Hemodialysis, } \\
\text { Liver biopsy ( } 1 \mathrm{Pt} .)\end{array}$ & Improved \\
\hline Lee [15] (1996) & 1 & - & Amanita virosa & 7 & GI symptoms & Liver failure & & Improved \\
\hline Yoo [16] 998) & 22 & Gyeongbuk & Amanita genus & 10 & $\begin{array}{l}\text { GI symptoms, Altered } \\
\text { mental status ( } 8 \text { Pts.), } \\
\text { Seizures ( } 1 \mathrm{Pt} .)\end{array}$ & $\begin{array}{l}\text { Liver failure (17 Pts.), } \\
\text { Renal failure (7 Pts.), } \\
\text { Pancreatitis (1 Pt.) }\end{array}$ & Hemodialysis (1) & 6 deaths \\
\hline Jung [17] (1999) & 2 & Busan & Amanita virosa & 12 & GI symptoms & Renal failure & Renal biopsy (1 Pt.) & All improved \\
\hline Ahn [6] (2000) & 43 & $\begin{array}{l}\text { Western Gangwon } \\
\text { and Northern } \\
\text { Gyeongbuk }\end{array}$ & $\begin{array}{l}\text { Amanitar virosa } \\
\text { and Amanita } \\
\text { subjunquillea, } \\
\text { Unknown (4 Pts.) }\end{array}$ & - & - & Coagulopathy & & 7 deaths \\
\hline Lim [18] (2000) & 3 & $\begin{array}{l}\text { Gangwon } \\
\text { (Samcheok) }\end{array}$ & Amanita virosa & 11 & $\begin{array}{l}\text { GI symptoms, Seizures } \\
\text { (1 Pt.) }\end{array}$ & $\begin{array}{l}\text { Liver failure, Renal } \\
\text { failure, Coagulopathy ( } 2 \\
\text { Pts.), Encephalopathy ( } 2 \\
\text { Pts.) }\end{array}$ & $\begin{array}{l}\text { Charcoal intake, Fresh } \\
\text { frozen plasma }\end{array}$ & $\begin{array}{l}2 \text { improved, } 1 \\
\text { death }\end{array}$ \\
\hline $\operatorname{Kim}[19](2000)$ & 16 & Gyeongbuk (14 Pts.) & $\begin{array}{l}\text { Amanita virosa } \\
(2 \text { Pts. }) \text { Amanita } \\
\text { subjunquillea, } \quad(8 \\
\text { Pts. })\end{array}$ & 11 & GI symptoms & Liver failure & & 3 deaths \\
\hline Rho [20] (2000) & 16 & Gyeongbuk & $\begin{array}{l}\text { Amanita } \\
\text { subjunquillea }(1 \\
\text { Pt. with Amanita } \\
\text { virosa })\end{array}$ & $3 \sim 17$ & $\begin{array}{l}\text { GI symptoms, Altered } \\
\text { mental status ( } 2 \text { Pts.) }\end{array}$ & $\begin{array}{l}\text { Liver failure, Renal fail- } \\
\text { ure, Coagulopathy (13 } \\
\text { Pts.) }\end{array}$ & $\begin{array}{l}\text { Liver biopsy, Hemodial- } \\
\text { ysis }\end{array}$ & 2 deaths \\
\hline Jeong [21] (2003) & 3 & - & $\begin{array}{l}\text { Amanita subjunquil- } \\
\text { lea }\end{array}$ & - & GI symptoms & Liver failure & Liver biopsy & Improved \\
\hline Oh [22] (2004) & 19 & - & Amanita genus & 9 & GI symptoms & Renal failure (1 Pt) & & Improved \\
\hline Suh [23] (2006) & 4 & Chungbuk & Amanita genus & 11 & GI symptoms & Liver failure & & Improved \\
\hline Moon [8] (2010) & 1 & Gyeonggi & Amanita virgineoides & 11 & GI symptoms, Anuria & Fulminant liver failure & Charcoal intake & Improved \\
\hline Kang [24] (2015) & 1 & Southern Gyeonggi & Amanita punctata & & Nausea, Dizziness & Renal failure & Renal biopsy & Improved \\
\hline
\end{tabular}


In the detailed full-text review, we investigated baseline sample characteristics, including number of patients, geographic region, mushroom subtype, incubation period, signs and symptoms presented in early and late stages, treatment course, and outcomes. Early-stage gastrointestinal (GI) symptoms included nausea, vomiting, watery diarrhea or abdominal pain, and altered mental status (classified as drowsiness, stupor, semicoma, or coma). Seizures and altered mental status were considered as severe CNS symptoms.

\subsection{Assessment of methodological quality}

The methodological quality of the identified studies was assessed using an 18-item validated quality appraisal tool for case series $[9,10]$.

\subsection{Statistical analysis}

Meta-analysis was performed using Review Manager software version 5.3 (RevMan; The Cochrane Collaboration 2012, The Nordic Cochrane Center, Copenhagen, Denmark). We extracted the number of patients with early-stage severe CNS symptoms and the frequency of mortality after treatment, and then conducted a meta-analysis of studies that included quantifiable outcomes. We employed the dichotomous method in RevMan to estimate the prognostic effect using the risk ratio $(R R)$ and risk difference $(R D)$ and their $95 \%$ confidence intervals (CIs). Heterogeneity in each analysis was quantified using the $\mathrm{I}^{2}$ statistic.

\section{Results}

\subsection{Search result and study selection}

The literature search yielded 1,539 articles in Embase, 1,706 in MEDLINE, 1,265 in Web of Science, 57 in KoreaMED, and 514 in Korean Studies Information System. After excluding duplicate references, 3,999 studies remained, of which 3,936 were deemed irrelevant to the review. After performing a fulltext review of the remaining 63 studies, 16 studies met the inclusion criteria for the review [6, 8, 11-24], and five studies were included in the meta-analysis (Fig. 1) [12, 16, 17, 20, 22].

\subsection{Type of mushroom and generation region}

The characteristics of the included studies are shown in Table 1. Eight studies reported on Amanita virosa poisoning, and the remaining studies reported poisoning by Amanita subjunquillea, Amanita virgineoides, and Amanita punctate. Most of the cases occurred in Kyungbuk and Kangwon in the 1990s or early 2000s (Fig. 2). In Kyunggi, the recent episodes of poisoning were reported between 2009 and 2015. The overall mortality rate was $16.5 \%$ (15 deaths of 91 patients).

\subsection{Clinical features, evaluation, and treatment}

A total of 146 patients from five studies were included in the meta-analysis, with an overall mortality rate of $17.8 \%$. Symptoms developed between 2 and $17 \mathrm{~h}$ after ingestion of mushrooms and included GI problems, such as nausea, vomiting, and watery diarrhea (Table 2). In some cases, additional CNS symptoms, such as altered mental status or seizures, developed and late-stage oliguria was also reported. Some patients progressed to liver failure with elevated aspartate transaminase (AST) and alanine transaminase (ALT), or to late-stage renal failure. Other symptoms included decreased platelet count and coagulopathy.

TA B L E 2. Complications of amatoxin poisoning

\begin{tabular}{|lc|}
\hline Symptoms & Complications \\
\hline GI symptoms & Liver failure \\
\hline Nausea & Renal failure \\
\hline Vomiting & Coagulopathy \\
\hline Watery diarrhea & Encephalopathy \\
\hline Abdominal pain & Pancreatitis \\
\hline CNS symptoms & \\
\hline Altered mental status & \\
\hline Seizures & \\
\hline Anuria, oliguria & \\
\hline Jaundice & \\
\hline Dizziness, headache & \\
\hline
\end{tabular}

Most patients received conservative treatment, including fluid therapy and charcoal intake. If the elapsed time after ingestion was short, gastric lavage was performed. Hemodialysis was performed for patients whose condition had progressed to renal failure. Liver or renal biopsies were also performed, if necessary.

\subsection{Quality assessment of the included studies and a meta-analysis of severe early-stage CNS symptoms and mortality}

Quality assessment showed that four of the five studies fulfilled more than $60 \%$ of the checklist criteria (See also Table S2). The RR for mortality associated with severe early-stage CNS symptoms was $10.56(95 \% \mathrm{CI}=2.73-40.83)$, and the $\mathrm{RD}$ was $0.57(95 \% \mathrm{CI}=0.21-0.81)$ (Table 3$)$.

\section{Discussion}

When patients visit clinics after intake of poisonous mushrooms, it is often difficult for clinicians to recognize toxinrelated symptoms. This is because of the long incubation period in which external signs and symptoms are absent that can often delay visiting the emergency room, as patients may assume that GI symptoms arise from less severe food poisoning $[1,25]$. As injury to target organs caused by mushroom toxins proceeds without the progression of external signs or symptoms, clinicians may miss the opportunity to reduce the toxin levels with interventions, such as gastric lavage or charcoal intake. In such cases, the clinical course may progress rapidly to hepatic or renal failure $[1,7,26]$. Despite adequate treatment, mushroom poisoning can lead to death, and the overall mortality rate owing to amatoxin-containing mushroom 


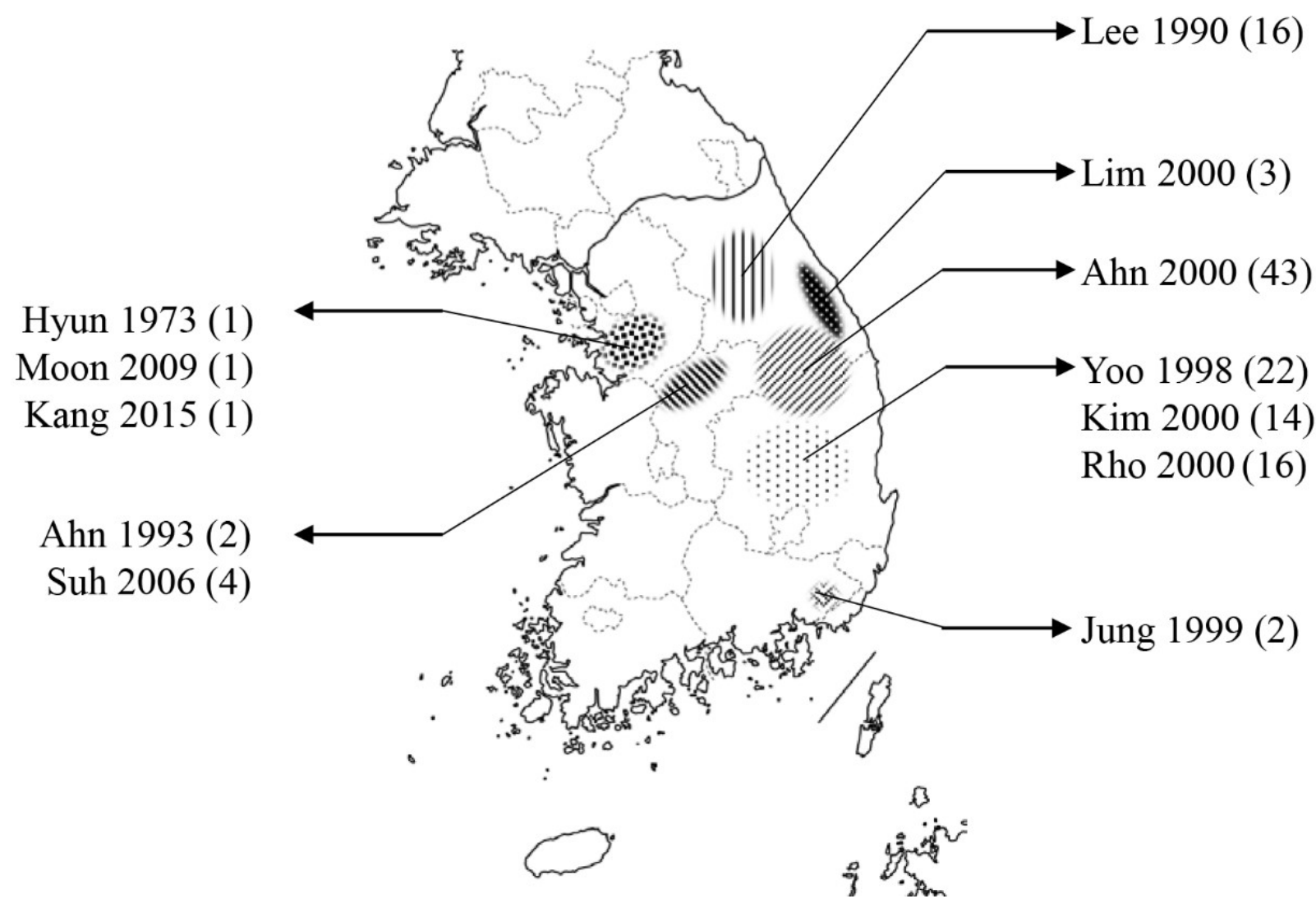

F I G U RE 2. A generating region of amatoxin poisoning in Korea.

poisoning was $16.5 \%$ in South Korea in this study.

Mushrooms are generally colorful, making them easy to avoid; however, some mushrooms are less conspicuous. Accidental intake of poisonous mushrooms occurs regularly because Amanita mushrooms appear similar to edible mushrooms (Fig. 3) [27, 28]. The typical shape of Amanita mushrooms includes an annulus structure with a volar shape underneath [29]. Poisonous mushrooms grow quickly during the rainy season [30], which occurs between late summer and autumn in South Korea. This period overlaps with the period in which edible mushrooms grow, leading to frequent intake of poisonous mushrooms [6]. The literature we reviewed reported intake of poisonous mushrooms in Kangwon and Kyungbuk, which are mountainous regions (Fig. 2). These areas were predominantly represented in the poisoning incidents that occurred in the late 1990s and early 2000s [6, 16, 19, 20]. Some cases occurred around the areas of Chungbuk and Kyunggi; however, no cases around Jeolla were reported, which consists of almost completely flat area. When mushrooms are gathered for food in an area where poisonous mushrooms grow, extra care is needed to avoid poisoning. Additionally, patients with GI signs or symptoms presenting in an area with frequent occurrence of mushroom poisoning should be administered a detailed questionnaire including the history of mushroom intake, the genus and species of mushrooms if patients have eaten poisonous mushrooms, and clinicians must closely monitor these patients after the first appearance of signs or symptoms.

Amatoxins are a group of bicyclic octapeptides occurring in some Amanita, Galerina and Lepiota species. As selective inhibitors of ribonucleic acid polymerase II, these compounds cause altered cell metabolism, reduced protein synthesis, and cell death [31]. After the intake of poisonous mushrooms, the toxin is absorbed and it affects the target organs involved in protein synthesis. The signs and symptoms of poisoning develop in stages [32]. In the early stage, GI symptoms, such as watery diarrhea, vomiting, nausea, and abdominal pain develop.

These symptoms are caused by the destruction of the mucosa by toxins. The incubation period is generally around 12 $\mathrm{h}$; however, it may vary among individuals. When eating a "GI irritant" a person primarily develops GI symptoms. However, individuals should be observed closely or admitted with continuous monitoring to detect the intake of amatoxincontaining mushrooms [1, 33]. When the toxin is absorbed through the bowel mucosa, it destroys the liver tissue, which generally occurs between 48 and $72 \mathrm{~h}$ after ingestion [4, 6, 7]. In these cases, liver biopsies reveal bleeding of the central vein, coagulation and necrosis of the central lobule, infiltration of inflammatory cells, or tissue fibrosis [14, 20, 34]. This phase is asymptomatic, as the early-stage GI symptoms improve. This can be a factor in delaying hospital admission [1, 6], even when liver failure proceeds asymptomatically. Subsequent symptoms include jaundice, encephalopathy, and altered mental status with elevated AST and ALT levels. In severe cases, cardiovascular impairment or multi-organ failure can progress rapidly, leading to death $[1,29,32]$.

Although acute renal failure may occur after hepatic failure, renal dysfunction occasionally develops during the early stages of poisoning [20]. Coagulopathy can also occur and may require infusion of fresh frozen plasma [18]. Thrombocytopenia 
TA B L E 3. Meta-analysis of significant CNS symptoms influencing survival in amatoxin poisoning

\begin{tabular}{|c|c|c|c|c|}
\hline \multirow[t]{2}{*}{ Study $^{a}$} & \multicolumn{2}{|c|}{ CNS symptoms ${ }^{b}$} & \multicolumn{2}{|c|}{ No CNS symptoms } \\
\hline & Death, n & Total, $\mathbf{n}$ & Death, n & Total, n \\
\hline Hyun 1973 [11] & 1 & 1 & 0 & 0 \\
\hline Lee 1990 [12] & 4 & 7 & 1 & 8 \\
\hline Lee 1990 [13] & 0 & 0 & 0 & 1 \\
\hline Ahn 1993 [14] & 0 & 0 & 0 & 2 \\
\hline Lee 1996 [15] & 0 & 0 & 0 & 1 \\
\hline Yoo 1998 [16] & 6 & 9 & 0 & 13 \\
\hline Jung 1999 [17] & 0 & 1 & 0 & 1 \\
\hline Lim 2000 [18] & 0 & 0 & 1 & 3 \\
\hline Rho 2000 [20] & 2 & 2 & 0 & 14 \\
\hline Jeong 2003 [21] & 0 & 0 & 0 & 3 \\
\hline Oh 2004 [22] & 0 & 1 & 0 & 18 \\
\hline Suh 2006 [23] & 0 & 0 & 0 & 4 \\
\hline Moon 2010 [8] & 0 & 0 & 0 & 1 \\
\hline Kang 2015 [24] & 0 & 0 & 0 & 1 \\
\hline Total, 95\% CI & & 21 & & 70 \\
\hline Total, death & 13 & & 2 & \\
\hline
\end{tabular}

Risk ratio of calculable studies in meta-analysis $[12,16,20]: \mathbf{1 0 . 5 6}[\mathbf{2 . 7 3 , 4 0 . 8 3}]$

Heterogenity: $\mathrm{Chi}^{2}=1.23, \mathrm{df}=2(P$ value 0.540$) ; \mathrm{I}^{2}=0 \%$

Test for overall effect: $Z=3.42(P$ value 0.001$)$

Risk difference of calculable studies in meta-analysis $[12,16,17,20,22]: \mathbf{0 . 5 7}[\mathbf{0 . 3 2}, \mathbf{0 . 8 1}]$

Heterogenity: $\mathrm{Chi}^{2}=9.65, \mathrm{df}=4(P$ value 0.05$) ; \mathrm{I}^{2}=59 \%$

Test for overall effect: $\mathrm{Z}=4.55(P$ value $<0.001)$

${ }^{a}$ We excluded studies in which frequency for death and CNS symptoms were not included.

${ }^{b}$ CNS symptoms included significant findings such as altered mental status and seizures.

[20], neuropathy [16], and skin erythema [15] can also develop, and some patients may present with pancreatitis [16]. Suh et $a l$. reported that the severity of metabolic acidosis during the therapeutic course could be related to the severity of hepatic failure [23].

The articles reviewed in this study revealed that the mean incubation period until the onset of symptoms in case of mushroom poisoning ranged between 2 and $17 \mathrm{~h}$, and GI symptoms, such as watery diarrhea, nausea, vomiting, and abdominal pain were presented in the early stages of poisoning. Some patients presented with symptoms of altered mental status, drowsiness, stupor, semicoma, or coma. Two patients experienced early-stage seizures. Severe CNS-related symptoms, such as seizures and altered mental status, may manifest as hepatic encephalopathy, 24 to $48 \mathrm{~h}$ after ingestion, during the stage of hepatic failure. We focused on severe CNS-related symptoms rather than the commonly known manifestations of the GI symptoms of poisonous mushrooms. When these symptoms manifest during the early stages of poisoning, they are associated with mortality, even after treatment. Of the 21 patients with initial CNS symptoms, 13 (61.9\%) did not survive. Our meta-analysis showed a strong association between early-onset
CNS symptoms and mortality in cases of amatoxin poisoning $(\mathrm{RR}=10.56 ; 95 \% \mathrm{CI}=2.73-40.83) . \quad$ Thus, severe earlystage CNS symptoms can be considered a strong risk factor for mortality.

CNS manifestations of mushroom poisoning are usually the consequence of hepatic failure, leading to hepatic encephalopathy, characterized by elevated blood ammonia levels in association with profound neurobehavioral changes [35]. Amatoxin-intoxicated patients with damaged liver function have difficulty in converting ammonia into urea, and the increased ammonia concentration in the brain can cause confusion, lethargy, encephalopathy, and convulsions [35, 36]. In several included studies, CNS symptoms were reported, which means that these symptoms may develop in response to abnormal liver and kidney functions.

Treatment for mushroom poisoning generally consists of conservative therapy, including fluid resuscitation, adjustment of electrolyte imbalance, proper supply of glucose, and administration of activated charcoal [1]. Although gastric lavage can be performed during the early stages of mushroom poisoning as needed, it may not be helpful if the stage of GI symptoms has already passed [37]. Repeated administration of activated 

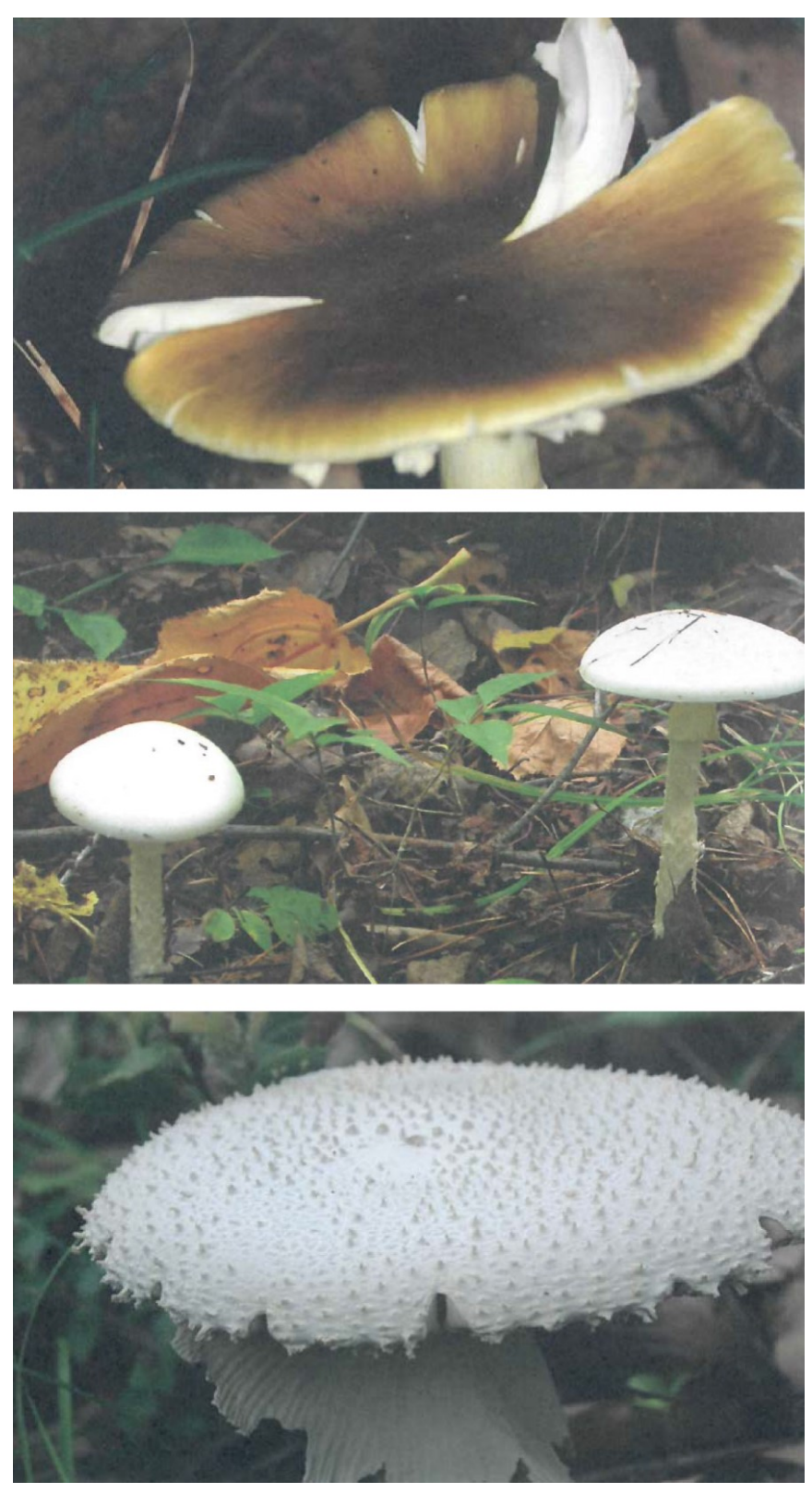

F I G URE 3. Amatoxin-containing mushrooms. (A) Amanita subjunquillea. (B) Amanita verna. (C) Amanita virosa. (Adapted with permission from reference 28. Copyright 2008, Gimmyoungsa).

charcoal inhibits the reabsorption of amatoxins. Suh et al. [20] reported repeated administration of activated charcoal during treatment [38]. When activated charcoal is used, it usually causes nausea and vomiting. Clinicians should be cautious in administration of activated charcoal, as it may cause pneumonia when aspirated. The goal of pharmacotherapy is to prevent liver damage, and various treatments are used. However, no therapy has been established as a standard practice. Penicillin $\mathrm{G}$ is commonly used to treat mushroom poisoning outside South Korea. Its use has also been reported in South Korea; however, evidence concerning its effectiveness is inconclusive $[23,39,40]$. $N$-acetylcysteine is used in the treatment of acute hepatic failure and improves the microcirculatory function of the liver; thus, decreasing mortality. $N$-acetylcysteine is especially effective in the treatment of acetaminophen poisoning, and is also used to treat hepatic failure caused by amatoxin poisoning. However, its precise effects in the treatment of amatoxin poisoning have not been established, and its use for this indication has been questioned [40]. Drugs, including silibinin and thioctic acid, have also been used for treatment [40, 41]. Evidence for silibinin efficacy is limited, but its use should be considered because of the few adverse effects and lack of alternative treatment options [41-43]. These drugs are the final treatment to consider to prevent irreversible liver damage $[40,44]$. Reversible liver damage can be improved through acute treatment with various drugs; however, patients who do not respond to these treatments can exhibit fatal outcomes, and liver transplantation may be required. Although many cases of liver transplantation have been reported, there have been relatively few in South Korea.

Our study had several limitations. First, although cases of accidental mushroom poisoning are rare, it is difficult to document all cases in South Korea. In particular, of the 27 cases of recent poisoning accidents occurring since 2004, only five studies have been published [45]. A unified mushroom poisoning reporting system needs to be established and standardized to support a systematic approach for case registration. Second, the majority of the included studies were case reports, and our meta-analysis based on these studies may have been biased. In addition, although we conducted a quality evaluation of the case reports, our interpretation was limited by the established quality evaluation for the meta-analysis. However, as no observational studies on mushroom poisoning have been conducted, and clinical trials would not be ethical, our analysis of existing case reports represents the most comprehensive analysis on this topic to date.

\section{Conclusions}

Amanita mushrooms are often mistaken for edible mushrooms, and their ingestion is frequently fatal. Since irreversible liver damage and renal failure can lead to a serious condition, which can then progress to multi-organ failure, rapid and active treatment is required. In addition, CNS manifestations, such as altered mental status and seizures, are usually the consequence of abnormal liver function, and patients with these symptoms at the early stage are associated with severe mortality risk.

\section{AUTHOR CONTRIBUTIONS}

CA and HK designed the review. CA and JO performed the searches and screened studies for eligibility. CA and HK assessed the quality of the papers and performed the statistical analysis. CA wrote the manuscript, and CA, THL and HK contributed to editorial changes in the manuscript. All authors read and approved the final manuscript.

\section{ACKNOWLEDGMENT}

The author would like to thank Dr. Sun Ja Seok for permitting us to use the images. 


\section{CONFLICT OF INTEREST}

The authors declare that there is no conflict of interest regarding the publication of this article.

\section{DATA AVAILABILITY}

The data used to support the findings of this study are available from the corresponding author upon request.

\section{SUPPLEMENTARY MATERIAL}

Supplementary material associated with this article can be found, in the online version, at https: //oss.signavitae.com/mre-signavitae/article/ $1395294407832289280 /$ attachment/SV2020120403_ Supplemental_Information.docx.

\section{REFERENCES}

[1] Nelson LS, Howland MA, Lewin NA, Smith SW, Goldfrank LR, Hoffman RS. Goldfrank's toxicologic emergencies. 11th ed. NY: McGraw-Hill Education. 2019.

[2] Berger KJ, Guss DA. Mycotoxins revisited: part I. The Journal of Emergency Medicine. 2005; 28: 53-62.

[3] Shannon M, Borron S and Burns M. Haddad and Winchester's clinical management of poisoning and drug overdose. 4th ed. Philadelphia: Saunders and Elsevier. 2007.

[4] Sohn CH. Type and treatment of toxic mushroom poisoning in Korea. Journal of the Korean Medical Association. 2015; 58: 818. (In Korean)

[5] Broussard C. Mushroom poisoning-from diarrhea to liver transplantation. The American Journal of Gastroenterology. 2001; 96: 3195-3198.

[6] Ahn BM, Lee DS, Lee KM, Kang SB, Yang JM, Park YM, et al. Amatoxins poisonings in Korea. The Korean Journal of Hepatology. 2000; 6: 340-349. (In Korean)

[7] Seok S, Kim Y, Kim W, et al. Encyclopedia of poisonous mushrooms. Purenhaengbok. 2011.

[8] Eun Joon M, M.D, Joo An H, M.D, Da Mi L, M.D, et al. A case of acute renal failure complicated by the poisoning of Amanita virgineoides. Kidney Research and Clinical Practice. 2010; 29: 140-143.

[9] Zeng X, Zhang Y, Kwong JSW, Zhang C, Li S, Sun F, et al. The methodological quality assessment tools for preclinical and clinical studies, systematic review and meta-analysis, and clinical practice guideline: a systematic review. Journal of Evidence-Based Medicine. 2015; 8: 2-10.

[10] Moga C, Guo B, Schopflocher D, Harstall C. Development of a quality appraisal tool for case series studies using a modified Delphi technique. Edmonton AB: Institute of Health Economics. 2012.

[11] Hong JS, Kim CW, Kim YS, Hyun JH. Acute yellow atrophy of the liver inducing mushroom poisoning. The Korean Journal of Medicine. 1973; 16: 147-153. (In Korean)

[12] Lee K, Lee J, Min B, Choi S, Jang W, Kwon SJ, et al. The 16 cases of fatal mushroom poisoning on 1987 in Young Seo Region. The Korean Journal of Internal Medicine. 1990; 38: 58-67. (In Korean)

[13] Lee SH, Cho HS, Hong UK, et al. A case of acute renal failure due to mushroom poisoning. Kidney Research and Clinical Practice. 1990; 9: 536-540. (In Korean)

[14] Ahn BM, Kim JI, Kim BW, et al. Two cases of Amanita mushroom poisonings. The Korean Journal of Gastroenterology. 1993; 25: 603-610. (In Korean)

[15] Lee KM, Won WH, Song SY, et al. A case of Amanita virosa intoxication. The Korean Journal of Gastroenterology. 1996; 28: 576-581. (In Korean)

[16] Yoo BD, Ahn YH, Choi WI, et al. Clinical analysis of the mushroom poisoning. Journal of Korean Society Emergency Medicine. 1998; 9: 323329. (In Korean)

[17] Hyun Chul J, Bo Suk K, Sang Heun S, Yong Bum K, Ho Jin S, Dong Won
$\mathrm{L}$, et al. Two cases of acute renal failure complicated by the poisoning of Amanita virosa. The Korean Journal of Medicine. 1999; 57: 1053-1057.

[18] Lim JG, Kim JH, Lee CY, Lee SI, Kim YS. Amanita virosa induced toxic hepatitis: report of three cases. Yonsei Medical Journal. 2000; 41: 416421.

[19] Kim KS, Lim YW, Jung JS, et al. Clinical analysis of phalloides syndrome. Journal of Korean American Medical Association. 2000; 11: 224-230. (In Korean)

[20] Rho HJ, Kim JH, Kang HR, et al. Clinical manifestations of Amanita subjunquillea poisoning. Korean Journal of Medicine. 2000; 58: 453 461. (In Korean)

[21] Jeong SY, Lee J, Bae JH, et al. 99mTc-DTPA galactosyl human serum albumin scintigraphy in mushroom poisoning patient: comparison with liver ultrasonography. Korean Journal of Nuclear Medicine. 2003; 37: 254-259. (In Korean)

[22] Oh B, Kim W and Lim K. Outcomes of acute liver injury from accidental mushroom poisoning. Journal of the Korean Society of Clinical Toxicology. 2004; 2: 116-122. (In Korean)

[23] Suh J-H, Kim S-J, Chung Y-K, Choi W-G, Kwon Y-S, Roh H-K, et al. Severe liver toxicity caused by amatoxin (case series). Journal of the Korean Society of Clinical Toxicology. 2006; 4: 73-77. (In Korean)

[24] Kang E, Cheong K, Lee M, Kim S, Shin G, Kim H, et al. Severe but reversible acute kidney injury resulting from Amanita punctata poisoning. Kidney Research and Clinical Practice. 2015; 34: 233-236.

[25] Ryu C, Lee M. Food poisoning. Journal of the Korean Medical Association. 2011; 54: 617. (In Korean)

[26] Garrouste C, Hémery M, Boudat AM, Kamar N. Amanita phalloides poisoning-induced end-stage renal failure. Clinical Nephrology. 2009; 71: $571-574$

[27] Kim S, Kim K and Suh G. A case of phalloides syndrome where T-PLS was used for hemodynamic support. Journal of the Korean Society of Clinical Toxicology. 2004; 2: 133-136. (In Korean)

[28] Rural development administration. The mushrooms of Korea: Gimmyoungsa. 2008; 367-373.

[29] Ward J, Kapadia K, Brush E, Salhanick SD. Amatoxin poisoning: case reports and review of current therapies. Journal of Emergency Medicine. 2013; 44: 116-121.

[30] Gürbüz S, Oguzturk H, Turgut K, Turtay MG, Guven T. Mushrooms: the velvety poison. Acta Medica Mediterranea. 2003; 31: 947-951.

[31] Karlson-Stiber C, Persson H. Cytotoxic fungi-an overview. Toxicon. 2003; 42: 339-349.

[32] Vetter J. Toxins of Amanita phalloides. Toxicon. 1998; 36: 13-24.

[33] Spoerke DG. Gastrointestinal irritant mushrooms (pp. 347-366). Handbook of mushroom poisoning-diagnosis and treatment. CRC Press. 1994

[34] Lefkowitch JH. The pathology of acute liver failure. Advances in Anatomic Pathology. 2016; 23: 144-158.

[35] Garcia J, Costa VM, Carvalho A, Baptista P, de Pinho PG, de Lourdes Bastos $\mathrm{M}$, et al. Amanita phalloides poisoning: mechanisms of toxicity and treatment. Food and Chemical Toxicology. 2015; 86: 41-55.

[36] Bonnet MS, Basson PW. The toxicology of Amanita phalloides. Homeopathy. 2002; 91: 249-254.

[37] Auerbach P, Cushing T, Harris NS. Wilderness medicine. 7th ed. Elsevier Mosby. 2016.

[38] Mas A. Mushrooms, amatoxins and the liver. Journal of Hepatology. 2005; 42: 166-169.

[39] Kwak BO, Bae SH. A case of hyperacute liver failure from mushroom intoxication in a child treated with penicillin. Korean Journal of Pediatric Gastroenterology and Nutrition. 2008; 11: 210. (In Korean)

[40] Enjalbert F, Rapior S, Nouguier-Soulé J, Guillon S, Amouroux N, Cabot C. Treatment of amatoxin poisoning: 20-year retrospective analysis. Journal of Toxicology Clinical Toxicology. 2002; 40: 715-757.

[41] Choi MW, Ko DR, Kong T, Choa MH, You JS, Chung SP, et al. Comparison of Silymarin, Penicillin, $N$-acetylcysteine in patient with amatoxin poisoning: a systematic review. Journal of the Korean Society of Clinical Toxicology. 2018; 16: 33-41.

[42] Lacombe $G$ and St-Onge $M$. Towards evidence-based emergency medicine: best BETs from the Manchester Royal Infirmary. BET 1: Silibinin in suspected amatoxin-containing mushroom poisoning. 2016; 33: 76-77.

[43] Ward J, Kapadia K, Brush E, Salhanick SD. Amatoxin poisoning: case 
reports and review of current therapies. Journal of Emergency Medicine. 2013; 44: 116-121.

[44] Ganzert M, Felgenhauer N, Zilker T. Indication of liver transplantation following amatoxin intoxication. Journal of Hepatology. 2005; 42: 202209.

[45] Korea Forest Research Institute, National Academy of Agricultural Science. Poisonous mushroom in the woods. Korea Forest Research Institute. 2014.
How to cite this article: Chiwon Ahn, Hyunggoo Kang, Tae Ho Lim, Jaehoon Oh. Poisoning due to ingestion of amatoxin-containing mushrooms in South Korea: a systematic review and meta-analysis. Signa Vitae. 2021;17(4):25-33. doi: $10.22514 /$ sv.2021.089. 